\title{
Hairy black holes sourced by a conformally coupled scalar field in $D$ dimensions
}

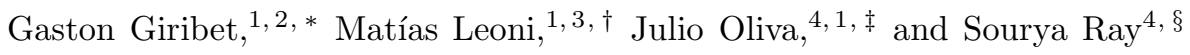 \\ ${ }^{1}$ Departamento de Física, Universidad de Buenos Aires FCEN-UBA, \\ IFIBA-CONICET, Ciudad Universitaria, Pabellón I, 1428, Buenos Aires, Argentina. \\ ${ }^{2}$ Instituto de Física, Pontificia Universidad Católica de Valparaíso, Casilla 4950, Valparaíso, Chile. \\ ${ }^{3}$ Instituto de Física de La Plata, Universidad Nacional de La Plata IFLP-UNLP, C.C. 67, 1900, La Plata, Argentina. \\ ${ }^{4}$ Instituto de Ciencias Físicas y Matemáticas, Universidad Austral de Chile, Valdivia, Chile
}

\begin{abstract}
There exist well-known no-hair theorems forbidding the existence of hairy black hole solutions in general relativity coupled to a scalar conformal field theory in asymptotically flat space. Even in the presence of cosmological constant, where no-hair theorems can usually be circumvented and black holes with conformal scalar hair were shown to exist in $D \leq 4$ dimensions, no-go results were reported for $D>4$. In this paper we prove that these obstructions can be evaded and we answer in the affirmative a question that remained open: Whether hairy black holes do exist in general relativity sourced by a conformally coupled scalar field in arbitrary dimensions. We find the analytic black hole solution in arbitrary dimension $D>4$, which exhibits a backreacting scalar hair that is regular everywhere outside and on the horizon. The metric asymptotes to (Anti-)de Sitter spacetime at large distance and admits spherical horizon as well as horizon of a different topology. We also find analytic solutions when higher-curvature corrections $\mathcal{O}\left(R^{n}\right)$ of arbitrary order $n$ are included in the gravity action.
\end{abstract}

PACS numbers: $11.10 . \mathrm{Kk}, 11.15 . \mathrm{Yc}, 11.25 . \mathrm{Hf}$

\section{INTRODUCTION}

There exist well-known no-hair theorems forbidding the existence of hairy black holes in general relativity (GR) conformally coupled to a scalar field theory, in asymptotically flat spacetime. More precisely, in the particular instance of a scalar field conformally coupled to GR with vanishing cosmological constant in $D=4$ spacetime dimensions black hole solutions do exist [2, 3], but they exhibit a scalar field configuration that diverges at the horizon. In dimension $D>4$, black hole solutions of this type simply do not exist at all [4, 5]. In the case of non-vanishing cosmological constant, in which the nohair theorems can usually be circumvented, black hole solutions with conformal scalar field configurations that are regular everywhere outside and on the horizon were found both in $D=3$ and $D=4$ dimensions 6 9]; however, the situation is quite different for $D>4$ where no-go results have been reported [10]. In this paper, we prove that these restrictions are circumvented if the scalar field theory is coupled to higher-order Euler densities in a conformally invariant manner. In this way, we answer in the affirmative a question that remained open: Whether hairy black holes do exist in GR sourced by a conformally coupled scalar field in arbitrary dimensions.

The paper is organized as follows: In section II we introduce the generalized conformal couplings. The theory obtained by adding such terms, which was introduced by

\footnotetext{
*Electronic address: gaston-at-df.uba.ar

$\dagger$ Electronic address: leoni-at-df.uba.ar

${ }^{\ddagger}$ Electronic address: julio.oliva-at-uach.cl

$\S$ Electronic address: ray-at-uach.cl
}

two of us in Ref. [1], is the most general theory of its sort yielding second order field equations, and hence is the natural extension of conformal couplings to higherdimensions. In section III we present a hairy black hole solution in GR in arbitrary dimensions $D>4$. The solution asymptote to locally (Anti-)de Sitter spacetime and admit spherical horizon as well as horizon of a different topology. In section IV we extend our solutions to the theory whose action also includes higher-order curvature terms $\mathcal{O}\left(R^{n}\right)$ for arbitrary $n$. For the latter theory, we also find analytic black hole solutions with a backreacting conformal scalar hair that is regular everywhere outside and on the horizon. We briefly comment on a duality symmetry that the theory exhibits and how it acts on the black hole solution.

\section{CONFORMAL FIELD THEORY}

We will be concerned with the special class of theory defined in Ref. [1], which consists of scalar matter conformally coupled to gravity through a non-minimal coupling between a real scalar field and the dimensionally extended Euler densities. This yields a theory whose equations of motion are of second order and presents quite interesting properties such as self-duality. In four dimensions this theory reduces to Einstein theory with a conformally coupled scalar field with potential $V(\varphi)=(\lambda / 4 !) \varphi^{4}$ and non-minimal coupling with the space-time curvature $-(1 / 12) R \varphi^{2}$. In higher dimensions, however, the theory admits more general couplings which can be conveniently written by defining a four-rank tensor $S^{\mu}{ }_{\nu \lambda \delta}$ made out of the Riemann curvature tensor $R^{\mu}{ }_{\nu \lambda \delta}$ and derivatives of 
the scalar field. More precisely, one defines

$$
\begin{gathered}
S_{\mu \nu}^{\gamma \delta}=\phi^{2} R_{\mu \nu}^{\gamma \delta}-4 \phi \delta_{[\mu}^{[\gamma} \nabla_{\nu]} \nabla^{\delta]} \phi+8 \delta_{[\mu}^{[\gamma} \nabla_{\nu]} \phi \nabla^{\delta]} \phi- \\
2 \delta_{[\mu}^{[\gamma} \delta_{\nu]}^{\delta]} \nabla_{\rho} \phi \nabla^{\rho} \phi
\end{gathered}
$$

which, indeed, can be seen to transform covariantly under local Weyl rescaling $g_{\mu \nu} \rightarrow \Omega^{2} g_{\mu \nu}$ and $\phi \rightarrow \Omega^{-1} \phi$. Note that, by construction, the tensor $S^{\mu}{ }_{\nu \lambda \delta}$ has the same algebraic symmetries as those of the Riemann tensor $R_{\nu \lambda \delta}^{\mu}$.

With tensor (11) one can easily write down the general action of the theory as

$$
I=\int d^{D} x \sqrt{-g} \sum_{k=0}^{\left[\frac{D-1}{2}\right]} \frac{1}{2^{k}} \delta^{(k)}\left(a_{k} R^{(k)}+b_{k} \phi^{D-4 k} S^{(k)}\right)
$$

where $\delta^{(0)} R^{(0)}=\delta^{(0)} S^{(0)}=1$, the symbol $\delta^{(k)}$ stands for the skew-symmetric Kronecker tensor

$$
\delta^{(k)}=k ! \delta_{\left[\alpha_{1}\right.}^{\mu_{1}} \delta_{\beta_{1}}^{\nu_{1}} \ldots \delta_{\alpha_{k}}^{\mu_{k}} \delta_{\left.\beta_{k}\right]}^{\nu_{k}},
$$

while for $k \geq 0, R^{(k)}$ and $S^{(k)}$ represent the $4 k$-rank tensors

$$
R^{(k)}=\prod_{r=1}^{k} R_{\mu_{r} \nu_{r}}^{\alpha_{r} \beta_{r}}, \quad S^{(k)}=\prod_{r=1}^{k} S_{\mu_{r} \nu_{r}}^{\alpha_{r} \beta_{r}} .
$$

$a_{k}$ and $b_{k}$ in (2) represent coupling constants that are in principle arbitrary. The theory will, however, exhibit special features for special relations between these constants.

The upper limit of the sum in (2) is the integer part of $(D-1) / 2$. When all the couplings $b_{k}$ in (2) vanish, the theory reduces to Lovelock theory of gravity and, in particular, if $a_{k \neq 1}=0$, it reduces to general relativity.

The field equations coming from (2) can be written as

$$
G_{\mu \nu}=T_{\mu \nu},
$$

where the symmetric tensors $G_{\mu \nu}$ and $T_{\mu \nu}$ are

$G_{\mu}^{\nu}=-\sum_{k=0}^{\left[\frac{D-1}{2}\right]} \frac{a_{k}}{2^{k+1}} \delta_{\mu \rho_{1} \ldots \rho_{2 k}}^{\nu \lambda_{1} \ldots \lambda_{2 k}} R_{\lambda_{1} \lambda_{2}}^{\rho_{1} \rho_{2}} \ldots R_{\lambda_{2 k-1} \lambda_{2 k}}^{\rho_{2 k-1} \rho_{2 k}}$,

$T_{\mu}^{\nu}=\sum_{k=0}^{\left[\frac{D-1}{2}\right]} \frac{b_{k}}{2^{k+1}} \phi^{D-4 k} \delta_{\mu \rho_{1} \ldots \rho_{2 k}}^{\nu \lambda_{1} \ldots \lambda_{2 k}} S_{\lambda_{1} \lambda_{2}}^{\rho_{1} \rho_{2}} \ldots S_{\lambda_{2 k-1} \lambda_{2 k}}^{\rho_{2 k-1} \rho_{2 k}}$.

In addition, the equation for the scalar field gives

$$
\sum_{k=0}^{\left[\frac{D-1}{2}\right]} \frac{(D-2 k) b_{k}}{2^{k}} \phi^{D-4 k-1} \delta^{(k)} S^{(k)}=0 .
$$

The theory defined by action (2) is the most general theory of gravity conformally coupled to a scalar field theory yielding second order field equations. As it should be for a conformal field with non-zero conformal weight, the trace of its associated energy-momentum tensor, $T_{\mu}^{\nu}$, vanishes on-shell (i.e. after Eq. (6) is imposed). To illustrate the structure of the action (2), let us write down the first terms explicitly: Up to terms that are linear in the Riemann tensor, the action reads

$$
\begin{gathered}
I=\int d^{D} x \sqrt{-g}\left(a_{0}+a_{1} R+b_{0} \phi^{D}+b_{1} \phi^{D-2}\right. \\
\left.\left(R+(D-1)(D-2) \phi^{-2}(\partial \phi)\right)^{2}\right)+\ldots
\end{gathered}
$$

Then, redefining the scalar field as $\phi \rightarrow \varphi=\phi^{(D-2) / 2}$, action (7) reduces to the familiar canonically normalized scalar conformal field theory

$$
\begin{gathered}
I=\int d^{D} x \sqrt{-g}\left(\frac{1}{16 \pi G} R-\frac{\Lambda}{8 \pi G}-\frac{1}{2}(\partial \varphi)^{2}-\right. \\
\left.\left.\frac{D-2}{8(D-1)} \varphi^{2} R-\frac{\lambda}{D !} \varphi^{\frac{2 D}{(D-2)}}\right)\right)+\ldots
\end{gathered}
$$

where we have conveniently denoted $a_{0} \equiv-\Lambda /(8 \pi G)$, $a_{1} \equiv 1 /(16 \pi G)$, and $b_{0} \equiv-\lambda / D$ !. The important feature here is that the conformal coupling $(\xi / 2) \varphi^{2} R$ with $\xi=-(D-2) /(4 D-4)$ appears automatically once the kinetic term for the field $\varphi$ is chosen to be canonically normalized, which in turn amounts to choosing $b_{1}=-(D-2) /(8(D-1))$. This is because both terms actually come from the same contribution $\phi^{D-4} S^{(1)}$ in (2).

The quadratic terms in the action take the form $\left(a_{2}+b_{2} \phi^{D-4}\right)\left(R^{2}-4 R_{\mu \nu} R^{\mu \nu}+R_{\mu \nu \lambda \delta} R^{\mu \nu \lambda \delta}\right)+\ldots$ where the ellipses stand for terms that contain up to second powers of the second derivative of $\phi$. This coupling between a real scalar field and the quadratic Gauss-Bonnet term resembles the next-to-leading contribution to the low energy effective action of string theory. The explicit form of the $S^{(2)}$ terms can be found in [1]. The complexity of the action increases notably ${ }^{1}$ with $k$.

For the theory (2) we will show that, remarkably, analytic hairy black hole solutions can be found in arbitrary dimension $D>4$. As a particular case we will find hairy black holes in GR coupled to scalar conformal field theory (CFT) in asymptotically (Anti-)de Sitter spacetime $((\mathrm{A}) \mathrm{dS})$. The existence of such solutions is remarkable because, as we discussed above, there are stringent nogo results reported in the search for conformal hairs in $D>4$. Our result shows which is the appropriate coupling needed for the black hole configurations to be supported by scalar conformal matter.

\footnotetext{
1 It can, however, be somewhat simplified by resorting to the first
} order formalism. 


\section{HAIRY BLACK HOLES}

Let us first consider GR coupled to the CFT defined by the matter content of action (2). That is, consider first the particular case $a_{k}=0$ for $k>1$ in (2). Up to quadratic terms in the matter part, the action reads

$$
\begin{gathered}
I=\int d^{D} x \sqrt{-g}\left(\frac{1}{16 \pi G} R-\frac{\Lambda}{8 \pi G}+b_{0} \phi^{D}+b_{1} \phi^{D-4} S+\right. \\
\left.b_{2} \phi^{D-8}\left(S_{\mu \nu \alpha \beta} S^{\mu \nu \alpha \beta}-4 S_{\mu \nu} S^{\mu \nu}+S^{2}\right)\right)+\ldots
\end{gathered}
$$

where $S_{\mu \nu}=S_{\mu \rho \nu}^{\rho}$ and $S=S_{\mu}^{\mu}$.

Generally, in $D$ dimensions one can include $S^{(k)}$ terms for $k=0,1,2, \ldots[(D-1) / 2]$ and there exist static black hole solutions, provided the couplings of such terms satisfy certain relations. Their metrics take the form

$$
d s^{2}=-f d t^{2}+f^{-1} d r^{2}+r^{2} d \Sigma_{D-2, \gamma}^{2}
$$

where $d \Sigma_{D-2, \gamma}^{2}$ is the metric of a $(D-2)$-dimensional Euclidean space of constant curvature $\gamma=(0, \pm 1)$ with radius one and volume $\operatorname{Vol}_{\Sigma}$, and where the metric function $f$ is given by

$$
\begin{gathered}
f(r)=\gamma-\frac{16 \pi G M}{(D-2) V o l_{\Sigma} r^{D-3}}-\frac{16 \pi G Q}{(D-1)(D-2) r^{D-2}}- \\
\frac{2 \Lambda}{(D-1)(D-2)} r^{2}
\end{gathered}
$$

where $a_{0}=-\Lambda /(8 \pi G), a_{1}=1 /(16 \pi G), M$ is an arbitrary constant ultimately associated to the mass of the solution, and $Q$ is given in terms of the couplings $b_{k}$ by the relation

$$
Q=\sum_{k=0}^{\left[\frac{D-1}{2}\right]}(D-2 k-1) \widetilde{b}_{k} \gamma^{k} N^{D-2 k}
$$

where $\widetilde{b}_{k}=b_{k}(D-1) ! /(D-2 k-1)$ !, and where $N$ is a dimensionful constant that appears in the scalar field configuration

$$
\phi(r)=\frac{N}{r}
$$

and satisfies the following constraints

$$
\begin{aligned}
\sum_{k=1}^{\left[\frac{D-1}{2}\right]} k \widetilde{b}_{k} \gamma^{k-1} N^{2-2 k} & =0, \\
\sum_{k=0}^{\left[\frac{D-1}{2}\right]}\left(D(D-1)+4 k^{2}\right) \widetilde{b}_{k} \gamma^{k} N^{-2 k} & =0 .
\end{aligned}
$$

The couplings $b_{k}$ have to obey the above constraints for the solution (10)-(11) to exist in $D>4$. This implies in particular, that in $D=5$ dimensions, for example, one needs to have $b_{0} \neq 0, b_{1} \neq 0$, and also $b_{2} \neq 0$.
Provided $\gamma \neq 0$ and the metric function $f(r)$ has least one positive root $^{2}$, solution (10)-(11) represents a hairy black hole with only one parameter, $M$. Indeed, the metric exhibits a scalar hair: The scalar field configuration (13) turns out to be regular everywhere outside and on the horizon; it only diverges at the origin, $r=0$, where the geometry develops a curvature singularity (cf. $3-$ 5]). The intensity of the scalar hair, given by $N$, cannot be changed and is governed by the quotients of coupling constants $b_{k}$. If the black hole has locally flat horizon $(\gamma=0)$, the hair disappears from the metric. In the case $\gamma= \pm 1$, in contrast, the scalar hair induces a contribution to the gravitational potential that damps off like $\sim Q / r^{(D-2)}$, which is consistent with the fact that matter is conformally coupled. In fact, the fall-off condition $\sim 1 / r^{D}$ is the expected one for the $T_{0}{ }^{0}(r)$ component of the CFT stress-tensor, which is the one that couples to gravity in the static spherically symmetric ansatz. Since the scalar field configuration is an actual hair in the sense that it falls off more rapidly than the Newtonian term $\sim M / r^{D-3}$, the solution happens to have a finite mass, which is given by $M$. The fall-off of $\phi \sim 1 / r$ in (13) in terms of the canonically normalized field translates into $\varphi \sim 1 / r^{(D-2) / 2}$. All these imply that the asymptotics remains locally (A)dS as in the non-hairy solution $\phi=0$, $Q=0$.

Solution (10)-(11) shares some properties with the $D=4$ black hole solution ${ }^{3}$ found in Ref. [8]. In the four-dimensional case, however, if $\Lambda<0$ then the only possible horizon geometry has negative constant curvature $(\gamma=-1)$, corresponding to a topological black hole with a Reissner-Nordström type contribution to the potential. In $D>4$ dimensions, in contrast, spherical horizons $(\gamma=1)$ are admitted and scalar CFT matter contributes differently relative to gauge fields.

There is a lesson to learn from our finding, which is the following: When trying to naively extend the $D \leq 4$ hairy black holes to $D>4$ dimensions, the solutions obtained do not exhibit a horizon but develop a naked singularity [10]. It turns out that if what one really wants is the horizon to persist together with a conformal scalar hair in $D>4$, then one has to add conformal couplings of the form $\varphi^{(2 D-4 k) /(D-2)} R^{(k)}+\ldots$ with $k>1$ and not only the usual term $\varphi^{2} R^{(1)}$; the latter is only sufficient in $D=3$ and $D=4$ where terms $S^{(k)}$ with $k>1$ do not contribute. The non-canonical kinetic terms for $\varphi$ coming from $S^{(k)}$ are also necessary, and this is the reason why

2 It is easy to verify that $f$ admits positive roots for special range of the parameter $M$ and coupling constants, so horizon does exist. For instance, consider the example in $D=5(k \leq 2)$, with $a_{0}>0$, $a_{1}>0(\Lambda<0), \gamma=1, b_{2}>0$ and $M$ large enough.

3 Nevertheless, it is worth pointing out that the solutions we found for $D>4$ do not exist in $D=4$. In this sense, our solutions are not a generalization of the $D=4$ solutions, but belong to a different class. It may be the case that there exists a larger set of solutions to theory (2) that would include both as special cases. 
previous attempts to find such a solution failed. Once one realizes this, the black hole solution found above can be easily generalized even to the case where $\mathcal{O}\left(R^{k}\right)$ terms for arbitrary $k$ are also included in the gravity action, i.e. to the case $a_{k \geq 2} \neq 0$. Let us discuss the general case in the next section.

\section{HIGHER-CURVATURE CORRECTIONS}

In $D$ dimensions, consider the action that includes both higher-curvature terms of the form $R^{(k)}$ in the gravity Lagrangian and terms of the form $S^{(k)}$ in the matter Lagrangian with $k>1$; namely consider the full action (2). It turns out that this generalized theory also admits the following solution

$$
d s^{2}=-f d t^{2}+f^{-1} d r^{2}+r^{2} d \Sigma_{D-2, \gamma}^{2},
$$

where, again, the scalar field configuration is given by (13), while the metric function $f$ now fulfills the polynomial equation

$$
\sum_{k=0}^{\left[\frac{D-1}{2}\right]} a_{k} \frac{(D-1) !}{(D-2 k-1) !}\left(\frac{\gamma-f(r)}{r^{2}}\right)^{k}=\frac{M(D-1)}{V o l_{\Sigma} r^{D-1}}+\frac{Q}{r^{D}}
$$

where $M$ is an arbitrary integration constant, and $Q$ is fixed to be

$$
Q=\sum_{k=0}^{\left[\frac{D-1}{2}\right]}(D-2 k-1) \widetilde{b}_{k} \gamma^{k} N^{D-2 k}
$$

As it happens in GR, constraints (14)-15) between couplings have to be imposed. Equations (14)-(15) fix the value of $N$ as well the relation between the couplings $b_{k}$ of the matter part.

Equation (17) is a polinomial of degree $k$ in $f$. It gives the solution for $f$, which can be explicitly expressed in terms of elementary operations only for $D<10$. Expression (17) is the analogue of Wheeler polynomial of Lovelock theory of pure gravity [11]. As in the GR case discussed in Section III, the solution has a single integration constant $M$, and for generic values of the couplings in the gravity action (i.e. $a_{k}$ being generic enough) there is always one Schwarzschild branch, for which $M$ turns out to be the mass of the solution. Constant $Q$ is fixed in terms of $N$ through (18), and the latter is fixed by (14)-(15). Therefore, the scalar field can be thought of as a secondary hair, as in the case in the three- and fourdimensional solutions previously reported in the literature.

\section{DISCUSSION}

In this paper we have proven that hairy black hole solutions in general relativity sourced by a conformally cou- pled scalar field do exist in arbitrary dimensions. This result provides an answer in the affirmative to a question that remained open and for which no-go results had been reported. The backreacting solutions we found here represent geometries that asymptote to (Anti-)de Sitter spacetime at large distances and admit both spherical horizons and horizons of a different topology. We have also shown how such black hole solutions extend to theories of gravity with higher-curvature corrections $\mathcal{O}\left(R^{n}\right)$ of arbitrary order $n$, for which we also found exact solutions.

Before concluding, let us comment that theory (2) exhibits other interesting properties such as self-duality. In fact, for special relations between couplings $a_{k}$ and $b_{k}$, the action of the theory is symmetric under the interchange of the pure gravity part of action (2) (i.e. the terms $R^{(k)}$ in $I$ ) with the matter part of it (i.e. the terms $S^{(k)}$ in $I$ ), accompanying this commutation with the inversion $\phi \rightarrow 1 / \phi$ of the scalar field. The symmetry under the interchange $R^{(k)} \leftrightarrow \phi^{D-4 k} S^{(k)}$ is clearly understood once one remembers that tensor $S_{\mu \nu}^{\gamma \delta}$ transforms covariantly under local Weyl transformations. In fact, this symmetry transformation can be realized by rescaling the metric as $g_{\mu \nu} \rightarrow \phi^{2} g_{\mu \nu}$. To gain intuition and see how this duality symmetry acts on the space of solutions of the theory one can consider the spherically symmetric solution (10)-(11), (13) derived above. Since, according to (13), $\phi=N / r$ and the duality transformation is given by the rescaling $g_{\mu \nu} \rightarrow \phi^{2} g_{\mu \nu} \sim r^{-2} g_{\mu \nu}$, one finds that the black hole metric gets transformed into a metric that is a direct product of a two-dimensional space with coordinates $(t, r)$ and the space of constant curvature $\Sigma_{D-2, \gamma}$. This duality symmetry and its action on the space of solutions of the theory will be studied in more detail in a future work. Let us just mention here that it corresponds to a type of ultraviolet/infrared correspondence which maps the large distance behavior of some solutions into the short distance limit ${ }^{4}$.

The authors thank Christos Charmousis and Mokhtar Hassaïne for many useful discussions. G. G. and J. O. thank the support of ICTP where part of this work was done. G. G. Also thanks Jorge Zanelli and the members of CECs, and specially thanks the Pontificia Universidad Católica de Valparaíso, where substantial part of this work was done. The work of G. G. and M. L. was supported by grants PIP, PICT, UBACyT, MathAmSud, BMWF-MINCyT, from CONICET, ANPCyT, and UBA. J. O. is supported by the FONDECYT Grant No. 1141073. S. R. is supported by FONDECYT Grant No. 11110176 and by CONICYT Grant No. 791100027. 
${ }^{4} \mathrm{Up}$ to terms $R^{(1)}$ and $S^{(1)}$ this correspondence has been explored in [12] for AdS wave solutions of the system in three dimensions.

[1] J. Oliva and S. Ray, Class. Quant. Grav. 29 (2012) 205008.

[2] N. Bocharova, K. Bronikov and V. Melnikov, Vestn. Mosk. Univ. Fiz. Astronom. 6 (1970) 706.

[3] D. Bekenstein, Ann. Phys. 82 (1974) 535; ibid 91 (1975) 72.

[4] B. Xanthopoulos and T. Dialynas, J. Math. Phys. 33 (1992) 1463.

[5] C. Klimčík, J. Math. Phys. 34 (1993) 1914.

[6] C. Martínez and J. Zanelli, Phys. Rev. D54 (1996) 3830.

[7] C. Martínez, J. P. Staforelli and R. Troncoso, Phys. Rev. D74 (2006) 044028.
[8] C. Martínez, R. Troncoso and J. Zanelli, Phys. Rev. D67 (2003) 024008.

[9] A. Anabalón and H. Maeda, Phys. Rev. D81 (2010) 041501.

[10] C. Martínez, "Black holes with a conformally coupled scalar field", in Quantum Mechanics of Fundamental Systems: The Quest for Beauty and Simplicity, M. Henneaux, J. Zanelli (eds.), Springer 2009.

[11] J.T. Wheeler, Nucl. Phys. B268 (1986) 737; ibid B273 (1986) 732.

[12] E. Ayón-Beato and M. Hassaïne, Phys. Rev. D73 (2006) 104001. 